\title{
Data variation analysis of QOS in mobile Adhoc network
}

\author{
Vindheyswari Prasad Tiwari”1 Ashish Xavier Das”2 Prof. A.K Jaiswal”3 \\ M-Tech Student, Adviser, co-Adviser Department of Electronic and Communication SHIATS, Allahabad, \\ India
}

\begin{abstract}
The proposed analysis is used to design a network according to the applications of mobile ad hoc network with different workloads. Network Services are considered end-to-end, this means from a Terminal Equipment (TE) to another TE. An End-to-End Service may have a certain Quality of Service (QoS) which is provided for the user of a network service. It is the user that decides whether he is satisfied with the provided QoS or not. This paper shows the variations of any parameter may affect the performance of ad hoc network. There are some metric for Quality of service ,Average end-to-end delay, Throughput and Average jitter, which are evaluated in this paper and analysis is also performed to show the effect of data variation.
\end{abstract}

Keyword: - Manets, DYMO, QOS, AODV

\section{INTRODUCTION}

A mobile ad hoc network (MANET) is a self-configuring infrastructure less network of mobile devices connected by wireless. Each device in a MANET is free to move independently in any direction, and will therefore change its links to other devices frequently. MANETs are a kind of wireless ad hoc networks that usually has a routable networking environment and autonomous collection of mobile users that communicate over relatively bandwidth constrained wireless links. Since the nodes are mobile, the network topology may change rapidly and unpredictably over time. The network is decentralized, where all network activity including discovering the topology and delivering messages must be executed by the nodes themselves, i.e., routing functionality will be incorporated into mobile nodes.

Protocols defined for ad hoc networks are classified as reactive protocols and proactive protocols. Reactive protocols are characterized by Mobile Networks (MNs) acquiring and maintaining routes on demand, while proactive protocols are characterized by all Mobile Networks maintaining routes to all destinations all the time. Examples of reactive protocols are DSR (Dynamic Source Routing), and AODV (Ad hoc on-demand Distance Vector). Examples of proactive protocols are OLSR (Optimized Link State Routing Protocol), and TBRPF (Topology Dissemination Based on Reverse-Path Forwarding). All these protocols have been analyzed and compared in several papers. The main conclusion on these comparisons is that none of them is the best for all environments. Depending on several aspects such as mobility, load of the network, diameter of the network, etc, a protocol may behave better than another. For obtaining QoS (Quality of Service) on a MANET, it is not sufficient to provide a basic routing functionality. Other aspects should also be taken into consideration such as bandwidth constraints due generally to a shared media, dynamic topology since Mobile Networks are mobile and the topology may change and power consumption due to limited batteries.

\section{DYMO PROTOCOL}

The Dynamic MANET On-demand (DYMO) routing protocol enables reactive, multi-hop routing between participating nodes that wish to communicate. The basic operations of the DYMO protocol are route discovery and management. During route discovery the originating node initiates dissemination of a Route Request (RREQ) throughout the network to find the target node. During this dissemination process, each intermediate node records a route to the originating node. When the target node receives the RREQ, it responds with a Route Reply (RREP) unicast toward the originating node. Each node that receives the RREP records a route to the target node, and then the RREP is unicast toward the originating node. When the originating node receives the RREP, routes have then been established between the originating node and the target node in both directions.

In order to react to changes in the network topology nodes maintain their routes and monitor their links. When a packet is received for a route that is no longer available the source of the packet is notified. A Route Error (RERR) is sent to the packet source to indicate the current route is broken. Once the source receives the RERR, it re-initiates route discovery if it still has packets to deliver. In order to enable extension of the base specification, DYMO defines a generic element structure and handling of future extensions. By 
defining a fixed structure and default handling, future extensions are handled in a predetermined fashion. DYMO uses sequence numbers as they have been proven to ensure loop freedom. Sequence numbers enable nodes to determine the order of DYMO route discovery packets, thereby avoiding use of stale routing information.

\section{RELATED WORK}

Routing problem in MANETs is a fundamental problem. In MANET group, four routing protocols have been standardized, including AODV, DSR, Optimized Link State Routing (OLSR) and Topology Dissemination Based on Reverse-Path Forwarding (TBRPF). Dynamic MANET On-demand (DYMO) Routing and OLSRv2 are two other routing protocols. Standard ad-hoc routing protocols can be divided into two categories: reactive (on-demand) and proactive. The on-demand routing protocols include AODV, DSR and DYMO. Reactive (On demand) routing protocols will flood route discovery messages upon arrival of a connection request. Proactive routing protocols require the some nodes to respond to any changes in network topology by broadcasting updates throughout the network. Proactive routing protocols include OLSR and TBRPF.

\section{SIMULATION}

In this paper we are evaluating various mobility speeds in Dynamic Manet on demand routing protocol (DYMO). This analysis done by NS2 simulator. The size of data are gradually varies for different workload with same networks while other parameters are constant.

A. Throughput: Throughput or network throughput is the average rate of successful message delivery over a communication channel. The throughput is usually measured in bits per second (bit/s or bps), and sometimes in data packets per second or data packets per time slot.

The amount of data that received through the network per unit time, i.e. data bytes delivered to their destinations per second

Throughput $=\underline{\text { Total byte received }}$

Total time

\section{B. End-to-end delay:}

End-to-end delay refers to the time taken for a packet to be transmitted across a network from source to destination. It represents the average data delay an application or a user experiences when transmitting data.

$\mathrm{d}_{\text {end-end }}=\mathrm{N}\left[\mathrm{d}_{\text {trans }}+\mathrm{d}_{\text {prop }}+\mathrm{d}_{\text {proc }}\right]$

Where

$\mathrm{d}_{\text {end-end }}=$ end-to-end delay

$\mathrm{d}_{\text {trans }}=$ transmission delay

$\mathrm{d}_{\text {prop }}=$ propagation delay

$\mathrm{d}_{\text {proc }}=$ processing delay

$\mathrm{N}=$ number of links (Number of routers +1 )

\section{Average Jitter:}

\section{Jitter is the time variation of a characteristic of the latency packets at the destination}

The simulation environment is designed as terrain size $1500 \mathrm{~m} 2$. The simulation time was set to 100 , 21 nodes placed in the terrain and the packet size is varies as 512, 1024 and 1536 bytes. The random way point mobility model is an entity mobility model (ranging the maximum speed of $10 \mathrm{mps}$ with the pause time of $30 \mathrm{~s}$ ) applied for the designed application traffic CBR [constant bit rate] and network used for multimedia streaming. The MAC protocol IEEE 802.11DCF (Distributed Co-ordination Function) which is suitable for MANETs was used where transmission range was set to $50 \mathrm{~m}$ and the data rate was $2 \mathrm{Mbps}$. The performance of the network carried out different workload and the variation in the data bytes.

\section{RESULT}

The figures shown below are the different parameter metrics of QOS of the proposed variation of the network. 


\section{Throughput}
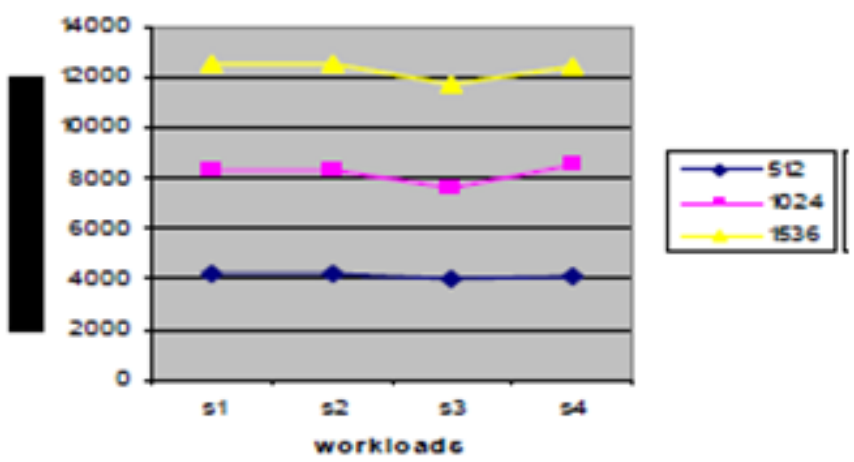

Fig 1. Throughput Analysis

Delay
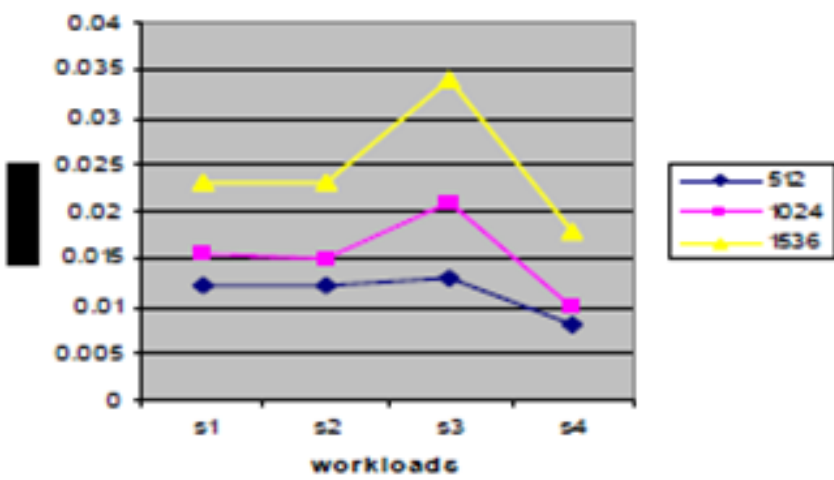

Fig 2. Delay Analysis

Jitter
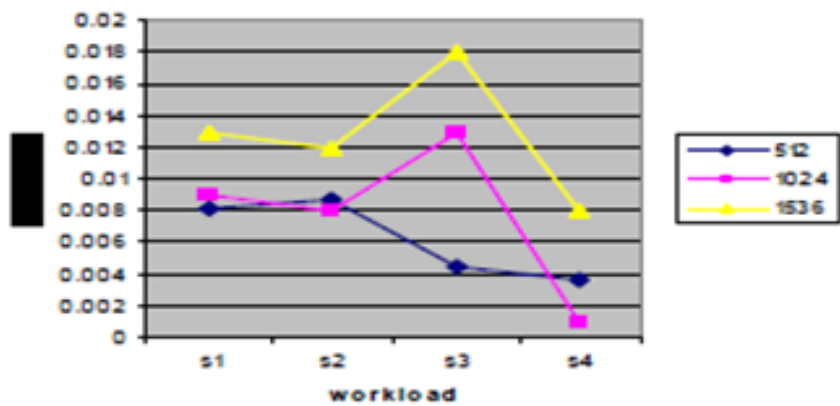

Fig 3. Jitter Analysis

[1] Johnson D. and Maltz D., Dynamic Source Routing in Ad-Hoc Wireless Networks. In Mobile Computing,

[2] Levis P. and Culler D. (2002). Mat'e: A Tiny Virtual Machine for Sensor Networks. In Proceedings of the 10th International Conference on Architectural Support for Programming Languages and Operating Systems (ASPLOS-X).

[3] Ma M. and Yang Y., (2007). "SenCar: an energy-efficient data gathering mechanism for large-scale multihop sensor networks," IEEE Trans. on Parallel and Distributed Syst, 18(10), 1476-1488.

[4] He L., Zhuang Y., Pan J. and Xu J., (2010). "Evaluating on-demand data collection with mobile elements in wireless sensor networks," in Proc. IEEE VTC'10-FALL.

[5] He L., Yang Z., Pan J. and Cai L., (2012) "Evaluating service disciplines for mobile elements in wireless ad hoc sensor networks," in PrOC. IEEE INFOCOM'12,.

[6] Stajano F. and Anderson R., (1999). "The resurrecting duckling: security issues for ad-hoc wireless networks," in Proceedings of the 7th International Workshop on Security Protocols, Cambridge, UK.

[7] Perkins C.E., Belding-Royer E.M. and Das S.R., (2003). "Ad Hoc On-Demand Distance Vector (AODV) Routing," IETF Mobile Ad Hoc Networks Working Group, Internet Draft, work in progress.

[8] Das S.R., Perkins C.E. and Belding-Royer E.M., (2000). "Performance Comparison of Two On-Demand Routing Protocols for Ad Hoc Networks," Proc. IEEE Infocom, vol. 1, 3-12. 